\title{
Exogenous ascorbic acid delayed leaf senescence of early flowering rice mutant FTL10
}

\author{
Q.L. ZHANG, W.Q. DING, Y.X. WEI, H. GAO, and C.L. PENG ${ }^{+}$ \\ Guangdong Provincial Key Laboratory of Biotechnology for Plant Development, School of Life Sciences, \\ South China Normal University, 510631 Guangzhou, China
}

\begin{abstract}
FTL10 is an early flowering mutant of OSFTL10-suppressed transgenic rice (Oryza sativa L.) with premature senescence phenotype. Early leaf senescence can cause negative effects on rice yield, therefore delaying leaf senescence and prolonging the leaf functional stage is one of the important approaches to increase the rice yield. It is well known that ascorbic acid (AsA) is involved in regulating plant growth. To explore the effect of AsA on leaf senescence of FTL10, we treated rice leaves with $0.28 \mathrm{mM}$ AsA. Results showed that total antioxidant capacity was higher and reactive oxygen species were lower in the AsA-treated group. The expression of senescence-associated genes was higher in the control group. Exogenous AsA can stabilize chlorophyll and Rubisco protein contents, delay leaf senescence, and maintain net photosynthetic rate $\left(P_{\mathrm{N}}\right)$ of rice leaves. Our results suggest that exogenous application of AsA can delay leaf senescence, increase $P_{\mathrm{N}}$, and then increase the rice yield.
\end{abstract}

Additional key words: nitroblue tetrazolium staining; nonenzymatic antioxidant; Rubisco large subunit; Rubisco small subunit; seed-setting rate; soluble protein.

\section{Introduction}

Rice (Oryza sativa L.) is one of the world most important crops and is the staple crop for approximately more than half the world's population (Iizumi and Ramankutty 2016). The yield is mainly provided by the photosynthesis of leaves after heading (Mae 1997). However, the grain yield of rice is often tightly linked to leaf senescence. Leaf senescence is considered the final stage of leaf development and is vital for plant fitness (Lim et al. 2007). It can be seen as an important phase in the plant life cycle (Nam 1997). Although senescence has great positive effects on plants, precocious senescence shortens the growth stage of crops and the functional period of leaves (Woo et al. 2013). Particularly in agricultural aspects, premature senescence results in poor grain quality, deterioration of the quality of vegetables, and reduction of crop yield (Jing and Nam 2012). It has been shown that premature senescence of flag leaves is negatively correlated with grain yield in rice (Zhang et al. 2007). The supply of assimilated carbon to grain at the grain-filling period can be enhanced by delaying leaf senescence, thereby enhancing the mass per grain (Jiang et al. 2007, Gregersen et al. 2013). Leaf senescence can be triggered by many endogenous and external factors (Lee et al. 2015). These factors include increased accumulation of ROS, such as hydrogen peroxide, superoxide, and hydroxyl radical (Van Breusegem and Dat 2006).

ROS, when accumulated at high concentrations, can oxidize the cellular AsA and glutathione pool of the cell (Bienert et al. 2006, Mittler et al. 2004). Previous study showed that endogenous ROS burst resulted in programmed cell death of starchy endosperm at $12 \mathrm{~d}$ after flowering $(\mathrm{Xu}$ et al. 2010). In another study, a higher accumulation of ROS in the rice leaves during the senescence period led to reduced yield (Zeng et al. 2018). Excessive ROS would destroy the photosynthetic apparatus (Hideg and Schreiber 2007); to control the ROS content, plants have developed enzymatic and nonenzymatic mechanisms. AsA is included in the nonenzymatic mechanisms (Gest et al. 2013).

AsA belongs to water-soluble antioxidants and is one of the most important vitamins widely distributed in various plants (Guerreiro et al. 2010). Some previous studies have demonstrated that AsA plays important roles in the regulation of mitosis and cell expansion (Smirnoff and Wheeler 2000), in electron transport chain (Tóth et al.

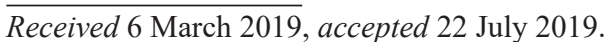

${ }^{+}$Corresponding author; phone: +86-20-85217612, fax:+86-20-85215535, e-mail: pengchl@scib.ac.cn

Abbreviations: AsA - ascorbic acid; Chl - chlorophyll; CK - normal temperature control group; DAB - diaminobenzidine; DAH - days after heading; DPPH - 1,1-diphenyl-2-picrylhydrazyl; FTL10 - OsFTL10-suppressed transgenic rice; NBT - nitroblue tetrazolium; $P_{\mathrm{N}}$ - net photosynthetic rate; $\mathrm{R}_{\mathrm{L}}-$ Rubisco large subunit; ROS - reactive oxygen species; $\mathrm{R}_{\mathrm{S}}$ - Rubisco small subunit; TAC - total antioxidant capacity; ZH-11 - wild-type.

Acknowledgements: The authors are very grateful to Professor Hongqing Li (South China Normal University, China) for the generous gift of rice seeds. This work was funded by Guangdong Province Natural Science Foundation (2015A030311023, 2017A030313167). The study was also supported by the National Natural Science Foundation of China $(31570398,31870374)$ and by the Innovation Project of Graduate School of South China Normal University. 
2009), and particularly, in scavenging of ROS (Ren et al. 2015). AsA is an important antioxidant of the ROS scavenging mechanism; it helps to reduce peroxides (Potters et al. 2010). AsA is considered one of the most abundant nonenzymatic antioxidants, which can regulate the content of ROS in higher plants (Gest et al. 2013). Meanwhile, AsA is involved in physiological processes, such as senescence regulation and stress response (Mekki et al. 2015, Chiang et al. 2017). It was reported that decreasing AsA content accelerated the senescence rate of tomato plants (Zhang et al. 2013). Recently, we have reported that rice plants with more AsA had a greater yield (Yu et al. 2015). The rice plants with insufficient AsA were sensitive to environmental factors and accumulated more ROS in the leaves (Yu et al. 2017, Zhang et al. 2018). ROS burst out after flowering in the rice and accelerate the senescence of leaves (Xu et al. 2010). The OsFTL10suppressed transgenic rice line FTL10 exhibited earlier flowering compared to wild type. This study aimed to explore the function of exogenous AsA in the process of senescence in rice line FTL10. The results suggest a close correlation between exogenous AsA and leaf senescence in FTL10. Exogenous AsA can effectively scavenge ROS, delay senescence, finally leading to the increased grain yield.

\section{Materials and methods}

Plant material: Oryza sativa L. cv. Zhonghua 11 (ZH-11), and an early flowering mutant FTL10 were used in this study (Fang et al. 2019). The pasteurized seeds were planted in an artificial climate incubator and grown under controlled conditions with an average temperature of $33^{\circ} \mathrm{C}$ light $/ 28^{\circ} \mathrm{C}$ dark, air humidity of $80 \%$, and a photoperiod of $14 \mathrm{~h}$ light $/ 10 \mathrm{~h}$ dark [PAR of $100 \mu \mathrm{mol}$ (photon) $\mathrm{m}^{-2} \mathrm{~s}^{-1}$ ]. As the seedlings had four leaves they were taken out gently and then transferred to the field. Flag leaves of FTL10 were sampled at 10:30-11:00 h at 0, 10, 20, 30, and $40 \mathrm{~d}$ after heading (DAH), and all sampled leaves were stored at $-80^{\circ} \mathrm{C}$ until further analysis.

Exogenous AsA: In the preliminary experiment, we set five exogenous AsA concentrations, 0.07, 0.14, 0.21, 0.28, and $0.35 \mathrm{mM}$, respectively. Preliminary experiment results showed that exogenous AsA of $0.28 \mathrm{mM}$ could significantly delay the senescence of early flowering mutant FTL10. FTL10 rice plants grown in the field were sprayed with exogenous AsA $(0.28 \mathrm{mM})$ solution at $12: 00$ h every day from 0 to $40 \mathrm{DAH}$ until the leaves were thoroughly wet. Control groups (CK) were sprayed with an equal volume of distilled water.

Detection of $\mathrm{H}_{2} \mathrm{O}_{2}$ and $\mathrm{O}_{2}{ }^{--}$in the tissue: $\mathrm{H}_{2} \mathrm{O}_{2}$ generation in leaves was detected by an endogenous peroxidasedependent in situ histochemical staining procedure using 3,3'-diaminobenzidine (DAB). Leaves were placed in a solution containing $1 \mathrm{mg}(\mathrm{DAB}) \mathrm{mL}^{-1}(\mathrm{pH} \mathrm{5.5)}$ for $2 \mathrm{~h}$ at room temperature after a brief vacuum infiltration for three times (10 min each). Oxidation of DAB leads to its polymerization and deposition at the site of $\mathrm{H}_{2} \mathrm{O}_{2}$ generation. The leaves were boiled in ethanol $(95 \%, \mathrm{v} / \mathrm{v})$ for $10 \mathrm{~min}$ and then stored in $60 \%$ glycerol. $\mathrm{H}_{2} \mathrm{O}_{2}$ production is visualized as a reddish-brown coloration (Liu et al. 2007).

$\mathrm{O}_{2}{ }^{--}$production was detected by nitroblue tetrazolium (NBT) staining. Leaves were placed in a solution containing NBT $\left(1 \mathrm{mg} \mathrm{mL}^{-1}\right)$ plus $\mathrm{NaN}_{3}(10 \mathrm{mM})$ solution in $10 \mathrm{mM}$ potassium phosphate buffer $(\mathrm{pH}$ 7.8). After vacuum infiltration for three times (10 min each), the leaves were stained for $4 \mathrm{~h}$ at room temperature. The leaves were then boiled in ethanol $(95 \%, \mathrm{v} / \mathrm{v})$ for $10 \mathrm{~min}$ and stored in $60 \%$ glycerol. The NBT was visualized as a dark blue-colored formazan deposit (Liu et al. 2007).

RNA isolation and quantitative RT-PCR: Total RNA was extracted from flag leaves using an Omega R6827 kit (Omega, GA, USA) in accordance with the manufacturer's instructions. First-strand cDNA was synthesized with oligo(dT) primers using a M-MLV reverse transcriptase kit (Takara, Kyoto, Japan), and then the cDNAs were stored at $-80^{\circ} \mathrm{C}$ until further analysis. Quantitative real-time PCR (q-PCR) was performed using an Applied Biosystems platform. Each $10 \mu \mathrm{L}$ of PCR reaction contained $0.5 \mu \mathrm{L}$ of cDNA, $3.5 \mu \mathrm{L}$ of RNAse free water, $5.2 \mu \mathrm{L}$ of SYBR $^{\circledR}$ Premix EX Tap ${ }^{\mathrm{TM}}$ II (Takara, Kyoto, Japan), and $0.4 \mu \mathrm{L}$ of each primer, which was synthesized by Invitrogen. Rice $O S U B Q$ was used as an internal control. Normalization and fold changes were calculated using the method of Livak and Schmittgen (2001). The primers for real-time PCR were designed as follows: 5'-CCAGGACAAGATGATCTGCC-3' (forward) and 5'-AAGAAGCTGAAGCATCCAGC-3' (reverse) for OsUBQ; 5'-ACCCGCAGCAGCTTCTCGCCAA-3' (forward) and 5'-CAAGCACCAAAACCTCCTCCAT-3' (reverse) for OsA12; 5'-AGGGGTGGTACAACAAGCTG-3' (forward) and 5'-GCTCCTTGCGGAAGATGTAG-3' (reverse) for OsSGR; 5'-GCACGGAGGCGAACGA-3' (forward) and 5'-TTGAGCGGTAGCACCCATT-3' (reverse) for Osh36; 5'-TATCTCAGATCATACCGGATG-3' (forward) and 5'-AAGTACTCCCTCAGCGATGG-3' (reverse) for OsFTL10; 5'-CAAGAAGCCGAACGGTTC-3' (forward) and 5'-GTTAGAGTGGAGCAGCAT-3' (reverse) for OSNAP.

Detection of TAC of leaves: TAC was evaluated by measuring the 1,1-diphenyl-2-picrylhydrazyl (DPPH) scavenging rate according to Saha et al. (2008). In brief, rice flag leaves $(0.2 \mathrm{~g})$ were ground with $2 \mathrm{~mL}$ of $95 \%$ methanol in an ice bath, and the homogenate was centrifuged at $13,000 \times \mathrm{g}$ at $4^{\circ} \mathrm{C}$ for $10 \mathrm{~min}$. The sample of $0.2 \mathrm{~mL}$ was added to $2.8 \mathrm{~mL}$ of $120 \mu \mathrm{M} \mathrm{DPPH}$ (dissolved in $95 \%$ methanol) for $5 \mathrm{~min}$ in the dark. The absorbance of mixture was measured at $517 \mathrm{~nm}(U V-2450$, Shimadzu, Kyoto, Japan). Different concentrations of DPPH were used as a standard. The free radical-scavenging capacity of the mixture was calculated according to the absorbance of the mixture at $517 \mathrm{~nm}$.

Measurement of $\mathrm{Chl}$ content: Fresh leaves $(0.1 \mathrm{~g})$ were extracted with $10 \mathrm{~mL}$ of $80 \%(\mathrm{v} / \mathrm{v})$ acetone at $4{ }^{\circ} \mathrm{C}$ for 
$24 \mathrm{~h}$. The absorbance of the extracts was measured at 663 and $645 \mathrm{~nm}$ using a UV-Vis 2450 spectrophotometer (Shimadzu, Tokyo, Japan). Chl $a$ and Chl $b$ were determined using the redetermined extinction coefficients and equations established by Lichtenthaler (1987): Chl $a$ $\left[\mathrm{mg} \mathrm{L}^{-1}\right]=12.21 \mathrm{~A}_{663}-2.81 \mathrm{~A}_{645}$ and $\mathrm{Chl} b\left[\mathrm{mg} \mathrm{L}^{-1}\right]=$ $20.13 \mathrm{~A}_{645}-5.03 \mathrm{~A}_{663}$.

Rubisco protein determination was carried out according to Liu et al. (2000). Fresh leaves of $0.1 \mathrm{~g}$ were homogenized in $2 \mathrm{~mL}$ of grinding medium composed of $50 \mathrm{mM}$ Tris- $\mathrm{HCl}$ (pH 7.8) buffer containing 5\% (w/v) polyvinylpyrrolidone (PVP), 0.1\% (v/v) $\beta$-mercaptoethanol, $0.1 \%$ (w/v) NaCl, $0.17 \%(\mathrm{w} / \mathrm{v})$ phenylmethanesulfonyl fluoride (PMSF), and $2 \%(\mathrm{v} / \mathrm{v})$ glycerol, and the homogenate was centrifuged at $13,000 \times g$ for $10 \mathrm{~min}$. Supernatant $(50 \mu \mathrm{L})$ was added to an equal volume of sample buffer sample buffer ( $\mathrm{pH}$ 7.6) composed of $10 \mathrm{mM}$ Tris, $2 \%(\mathrm{v} / \mathrm{v})$ 2-mercaptoethanol, $24 \%(\mathrm{v} / \mathrm{v})$ glycerol, $2 \%(\mathrm{w} / \mathrm{v})$ sodium dodecyl sulphate (SDS), and $0.02 \%(\mathrm{w} / \mathrm{v})$ bromophenol blue, and then incubated in boiling water for $5 \mathrm{~min}$. The samples $(10 \mu \mathrm{L})$ were loaded onto gels containing $12.5 \%(\mathrm{w} / \mathrm{v})$ resolving polyacrylamide gel $[12.1 \%$ acrylamide, $0.5 \mathrm{M}$ Tris- $\mathrm{HCl}$ (pH 8.8), 0.1\% SDS, 0.4\% N,N'-methylene-bis-acrylamide, $0.08 \%$ ammonium persulphate and $0.08 \%$ N,N,N',N'tetramethylethylene diamine (TEMED)] and 4\% (w/v) stacking polyacrylamide gel $[4 \%$ acrylamide, $0.125 \mathrm{M}$ Tris- $\mathrm{HCl}$ (pH 6.8), 0.1\% SDS, 0.1\% N,N'-methylene-bisacrylamide, $0.05 \%$ ammonium persulphate, and $0.08 \%$ TEMED]. Gel staining with Coomassie Brilliant Blue $R$-250 (Sigma, USA) was followed by destaining overnight with gentle shaking to make sure that its background turned colorless. The molecular mass of the Rubisco large $\left(\mathrm{R}_{\mathrm{L}}\right)$ and small subunit $\left(\mathrm{R}_{\mathrm{S}}\right)$ is 55 and $15 \mathrm{kDa}$, respectively. The SDS-PAGE gel was scanned using the GelDoc-It Imaging System (UVP, Upland, CA, USA) and relative grey value of each band detected by using TotalLab Quant software (TotalLab, Newcastle upon Tyne, UK). On the other hand, the supernatant was collected and diluted for 50 times. Then $250 \mu \mathrm{L}$ of diluted supernatant was added into the same volume of Bradford solution. After $5 \mathrm{~min}$, the absorbance was read at a wavelength of $595 \mathrm{~nm}$ (UV-Vis 2450 spectrophotometer, Shimadzu, Tokyo, Japan).

Measurement of $\boldsymbol{P}_{\mathrm{N}}$ : The $P_{\mathrm{N}}$ of flag leaves was determined at 10:00-11:30 h using the $L i-6400$ portable photosynthesis system (LI-COR, Inc., USA) on sunny days. The PPFD was set at $800 \mu \mathrm{mol} \mathrm{m} \mathrm{m}^{-2} \mathrm{~s}^{-1}$, the ratio of red and blue light of the irradiance in the leaf measurement chamber was set at 9:1, the corresponding mean temperature was $32 \pm 2{ }^{\circ} \mathrm{C}$, and the mean relative air humidity was $45 \%$. $P_{\mathrm{N}}$ was recorded when the values were relatively stable.

Yield and its component traits were also examined for FTL10 and ZH-11 plants, including mass of single spike, yield per plant, and seed set. Five plants in the middle of the inner two rows of each plot in the field were investigated to score the following traits for each plant: mass per panicle (mass in g of per panicle at $12-14 \%$ moisture), yield per plant (mass in $g$ of grains per plant at $12-14 \%$ moisture) and seed set (the number of filled grains per main panicle at maturity).

Statistical analysis: Values were expressed as means \pm standard error (SE). Statistical significance was determined by Student's $t$-test using SPSS Statistics 19.0 (IBM, NY, USA). Means were considered to be significantly different at the level $p<0.05$. Data were processed by using the SigmaPlot software (version 10.0, SYSTAT Software Inc., Richmond, CA, USA).

\section{Results}

The early flowering of FTL10: On the $50 \mathrm{~d}$ after transplanting, FTL10 has begun heading into reproductive growth and ZH-11 was still in the vegetative growth stage. FTL10 showed the phenotype of early flowering compared to $\mathrm{ZH}-11$. Leaf color was the most direct manifestation of rice senescence. Flag leaves of FTL10 were significantly more yellow than those of $\mathrm{ZH}-11$ at $80 \mathrm{~d}$ after transplanting, and it indicated that leaf senescence of FTL10 was faster than that of ZH-11 (Fig. 1D). The OsA12, OsSGR, and $O s h 36$ were all senescence-related genes in rice, and their

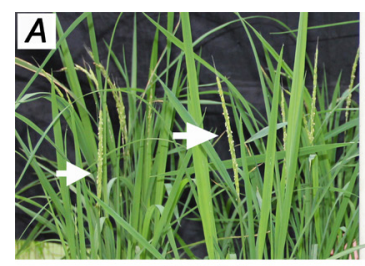

FTL10

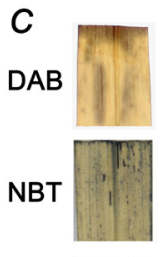

FTL10

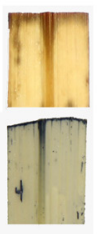

$\mathrm{ZH}-11$

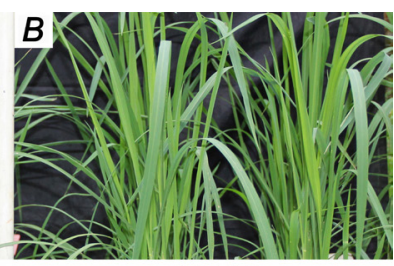

$\mathrm{ZH}-11$

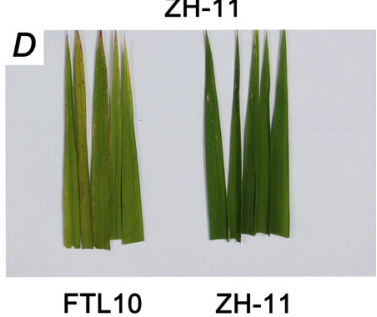

$\mathrm{ZH}-11$

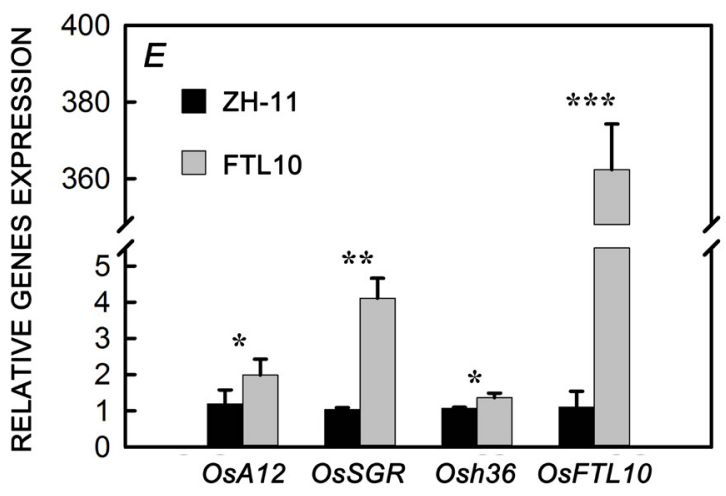

Fig. 1. The growth phenotypes of FTL10 $(A)$ and ZH-11 rice $(B)$, accumulation of $\mathrm{H}_{2} \mathrm{O}_{2}$ and $\mathrm{O}_{2}^{-}{ }^{-}$in the flag leaves visualized by 3,3'-diaminobenzidine (DAB) and nitroblue tetrazolium (NBT) staining, respectively $(C)$, senescence of rice leaves $(D)$, and relative genes expression $(E)$. Data are presented as the means \pm SE $(n=5)$. Asterisks indicate significant differences (two-sided Student's $t$-test, $\left.{ }^{*}-p<0.05,{ }^{*} *-p<0.01, * * *-p<0.001\right)$. 
expression was positively correlated with the senescence process of rice (Fig. $1 E$ ). At $80 \mathrm{~d}$ after transplanting, the expression levels of $O s A 12, O s S G R$, and $O s h 36$ genes in FTL10 were significantly 2 times, 4 times, and 1.2 times, respectively, higher than those in ZH-11. Expression of OsFTL10, an early flowering gene, was 360 times higher in FTL10 than that of ZH-11. The location of $\mathrm{H}_{2} \mathrm{O}_{2}$ and $\mathrm{O}_{2}{ }^{-}$ in flag leaves of rice $80 \mathrm{~d}$ after transplantation showed that the accumulation of $\mathrm{H}_{2} \mathrm{O}_{2}$ and $\mathrm{O}_{2}{ }^{-}$in leaves of FTL10 was higher than that of ZH-11 (Fig. $1 C$ ). The results suggested that early flowering resulted in early senescence.

Senescence of leaves: On the $40 \mathrm{DAH}$, there was a significant yellowing of the leaves without AsA treatment. AsA delayed the senescence of FTL10 leaves. Exogenous AsA could reduce the expression of senescence-related and early flowering genes (Fig. 2C). The expression levels of OSFTL10, OsNAP, and OSSGR genes decreased by $27.2,5.8$, and $33.1 \%$, respectively. The TAC of rice leaves increased gradually during senescence, and it was higher in the leaves after the AsA treatment than that of $\mathrm{CK}$ at all stages, suggesting that exogenous application of AsA could improve the TAC of leaves (Fig. $2 A$ ). ROS localization in rice leaves showed that the accumulation of $\mathrm{H}_{2} \mathrm{O}_{2}$ and $\mathrm{O}_{2}{ }^{-}$was higher in the leaves without AsA treatment, suggesting that the addition of exogenous AsA reduced the accumulation of $\mathrm{H}_{2} \mathrm{O}_{2}$ and $\mathrm{O}_{2}{ }^{-}$in leaves of FTL10 ((Fig. 2B).

Photosynthetic capacity: The Chl contents in leaves decreased gradually with heading time (Fig. $3 A$ ). It was higher in the leaves with AsA treatment compared with $\mathrm{CK}$ at all stages. On the $40 \mathrm{DAH}$, the content of Chl was $20.8 \%$ higher than that in CK. Degradation of soluble proteins is one of the basic characteristics of leaf senescence. The content of soluble protein decreased slowly and remained higher between 0 and $20 \mathrm{DAH}$, and then decreased fast. The application of exogenous antioxidant AsA effectively slowed down its degradation rate, so that the soluble protein content in the leaves with AsA treatment was higher than that of control group by $53.6 \%$ at $40 \mathrm{DAH}$ (Fig. $3 C$ ). At the same time, the $P_{\mathrm{N}}$ of leaves also gradually decreased. Exogenous application of AsA slowed down the decline of $P_{\mathrm{N}}$ in leaves (Fig. 3D). Rubisco, as the main enzyme of photosynthesis, was higher in the leaves with AsA treatment, and the $\mathrm{R}_{\mathrm{L}}$ and $\mathrm{R}_{\mathrm{S}}$ were 14.3 and $35.4 \%$, respectively, higher than that of CK at $40 \mathrm{DAH}$ (Fig. $3 B$ ).

Yield and grain phenotypes: The yield characteristics of FTL10 after $40 \mathrm{~d}$ of heading are shown in Fig. 4. Under natural aging conditions, the seed-setting rate of FTL10 was lower. The seed-setting rate could be significantly increased and the vacancy rate decreased by the application of exogenous AsA. Statistical analysis showed that the mass of single spike, yield per plant, and seed-setting rate of FTL10 in the control group were 88.5, 91.3, and 91.4\% of that in the AsA treatment group, respectively.

\section{Discussion}

Leaf senescence is a normal physiological phenomenon in rice, which has a very positive effect on rice itself, and is also necessary for the transport of organic matter from source to sink (Lim et al. 2007). However, premature senescence is unfavorable for the functional period of rice. In agricultural production, premature senescence of leaves shortens the functional period of leaves and thus reduces the rice yield. The FTL10 is an early flowering line by overexpressing the OsFTL10 gene (Fang et al. 2019). The expression level of OsFTL10 gene in FTL10 was significantly higher than that in the wild type. The results showed that the FTL10 was heading and flowering, while the wild type was still
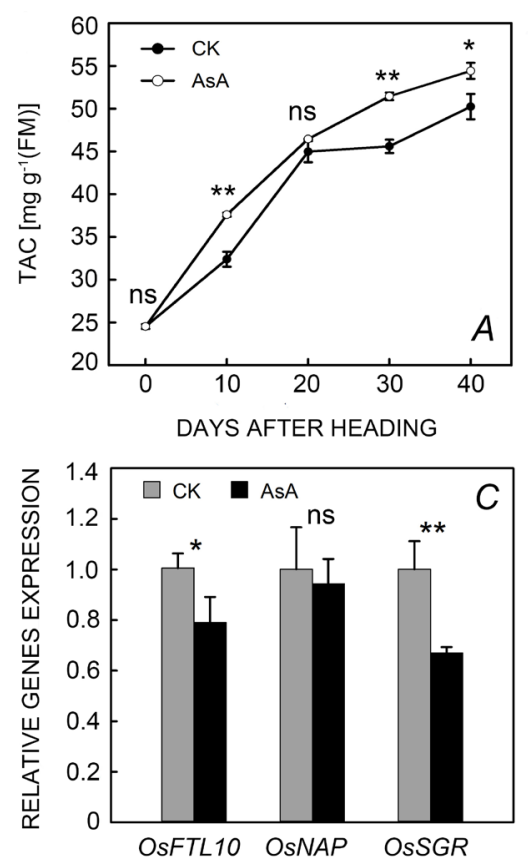

$B$

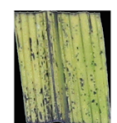

nivin

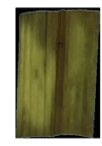

CK

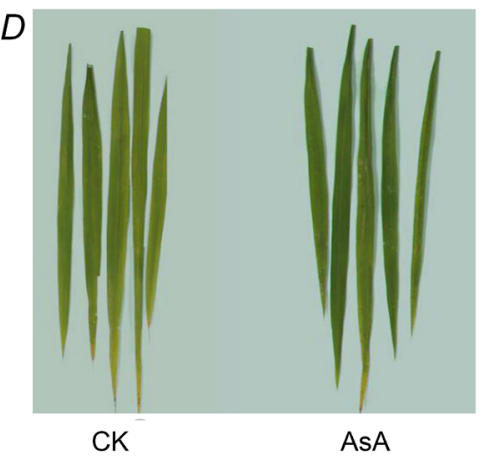

Fig. 2. Effect of ascorbic acid (AsA) treatment on total antioxidant capacity (TAC) of FTL10 rice leaves $(A)$, accumulation of $\mathrm{O}_{2}{ }^{-}$and $\mathrm{H}_{2} \mathrm{O}_{2}$ in the flag leaves visualized by nitroblue tetrazolium (NBT) and 3,3'-diaminobenzidine (DAB) staining, respectively $(B)$, relative genes expression $(C)$, and phenotypes of rice leaves $(D)$. Data are presented as the means $\pm \mathrm{SE}(n=5)$. Asterisks indicate significant differences (two-sided Student's $t$-test, $*_{-} p<0.05, * *-p<0.01, \mathrm{~ns}-$ not significant). 

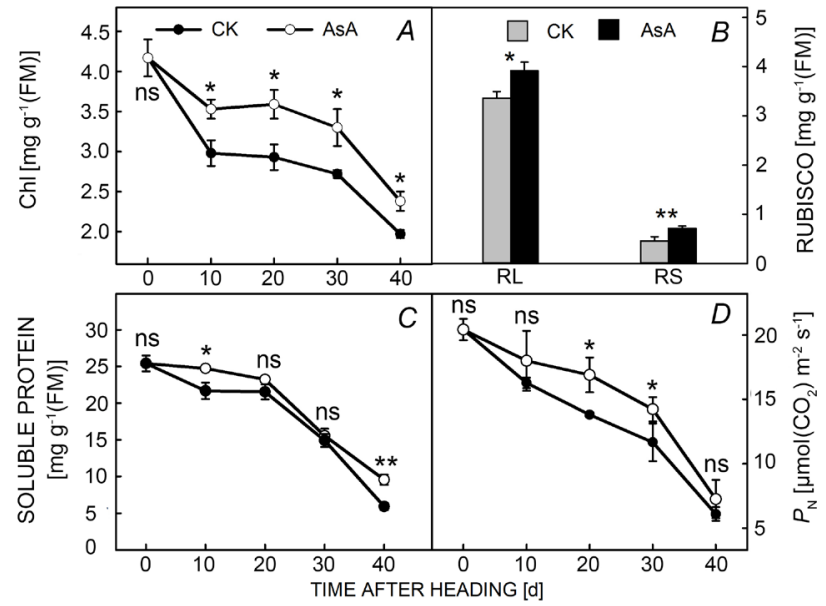

Fig. 3. Effect of ascorbic acid (AsA) treatment on chlorophyll (Chl) content $(A)$, Rubisco large $\left(\mathrm{R}_{\mathrm{L}}\right)$ and small subunit $\left(\mathrm{R}_{\mathrm{S}}\right)$ contents $40 \mathrm{~d}$ after heading $(B)$, soluble protein content $(C)$, and net photosynthetic rate $\left(P_{\mathrm{N}}\right)(D)$ of FTL10 rice leaves. Data are presented as the means $\pm \operatorname{SE}(n=5)$. Asterisks indicate significant differences (two-sided Student's $t$-test, ${ }^{*}-p<0.05,{ }^{*}{ }^{*}-p<0.01$, ns - not significant).
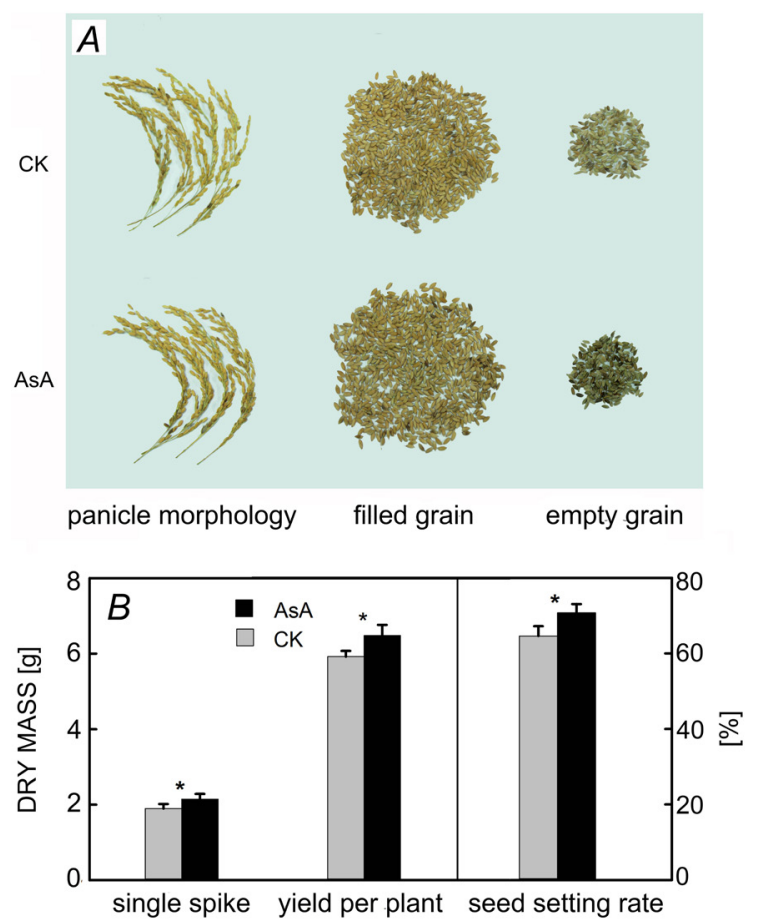

Fig. 4. Grain phenotypes $(A)$ and statistical results of mass of single spike, yield per plant, and seed-setting rate $(B)$ of FTL10 rice treated with ascorbic acid (AsA). Data are presented as the means $\pm \mathrm{SE}(n=5)$. Asterisks indicate significant differences (two-sided Student's $t$-test, $*-p<0.05$ ).

in the vegetative growth stage at $50 \mathrm{~d}$ after transplanting. The rice leaf phenotype showed that FTL10 was more senescent and it proved that early flowering rice shows the phenotype of premature senescence (Fig. 1). The balance between light energy capture and utilization was broken and it led to the formation of a large amount of ROS during the leaf senescence stage ( $\mathrm{Li}$ et al. 2009). It has been reported that the response of leaves to senescence signals is regulated by ROS (Van Breusegem and Dat 2006). At $80 \mathrm{~d}$ after transplanting, the accumulation of ROS in FTL10 was higher than that in the wild type and the expression of senescence-related genes was also higher (Fig. 1). This was consistent with previous observations in other rice cultivars (Gregersen et al. 2013).

AsA plays a very important role in plants metabolism and developmental growth (Alhagdow et al. 2007, Li et al. 2016), mediating the interaction of plants with the environment and oxidative stress (Gallie 2013). As one of the main antioxidants in plants, it can effectively scavenge ROS. In Arabidopsis, AsA deficiency leads to increased accumulation of ROS (Müller-Moulé et al. 2003). Our previous studies showed that the accumulation of ROS in grains was reduced by increasing the content of endogenous AsA (Yu et al. 2017). In this study, the application of exogenous AsA increased the TAC of leaves and reduced the accumulation of ROS (Fig. 2A,B). Low tissue AsA content is linked to premature senescence (Barth et al. 2004) and programmed cell death in plants (Pavet et al. 2005, De Pinto et al. 2012). At the same time, ROS can be used as signal substances in plants to cause plant senescence (Van Breusegem and Dat 2006). Previous studies have proved that AsA could regulate plant leaf senescence by controlling the content of ROS (Gallie 2013). On the $40 \mathrm{~d}$ after AsA treatment, the expression of OSFTL10, OSNAP, and OSSGR genes decreased significantly compared with $\mathrm{CK}$ (Fig. 2C). The OSSGR gene is involved in the disaggregation of Chl and the OSNAP gene is linked to the onset of leaf senescence (Liang et al. 2014). The phenotypes of leaves on the $40 \mathrm{~d}$ after AsA treatment showed that the leaves of the control group were more yellow (Fig. 2D). These results indicated that exogenous AsA can effectively scavenge ROS and then slow down leaf senescence.

Degradation of photosynthetic pigments and proteins is an important indicator of plant leaf senescence (Liang et al. 2014, Yang et al. 2016). With the senescence of rice leaves, ROS gradually accumulate. The $\mathrm{Chl}$ and soluble protein in leaves are gradually destroyed, resulting in a gradual decline of $P_{\mathrm{N}}$ in leaves (Fig. 3). Previous studies have found that different exogenous hormones can promote or slow down the degradation of Chl (Buchanan-Wollaston et al. 2005). In this study, the content of Chl was stabilized by applying exogenous AsA. Soluble protein, as a biological macromolecule, can be increased by increasing the expression of endogenous antioxidants. In the AsAtreated group, the soluble protein content was higher and Rubisco content was also higher. As the two most important substances of photosynthesis, Chl and Rubisco are closely related to $P_{\mathrm{N}}$, the degradation of them leads to the decline of $P_{\mathrm{N}}$. Our previous studies showed that the $P_{\mathrm{N}}$ of super high-yield rice was relatively high, and the $P_{\mathrm{N}}$ decreased slowly at the late growth stage (Ou et al. 2003). Therefore, maintaining the stability of photosynthetic apparatus during the rice senescence is the basis of yield. The $P_{\mathrm{N}}$ of the leaves treated with AsA was higher than that of the 
control group, and the yield was higher in the treatment group. There was a significant difference between the single pike mass, yield per plant, and seed-setting rate. In summary, our results suggest that exogenous AsA may play a role in delaying the senescence of rice leaves. It can effectively remove active oxygen, slow down the speed of leaf senescence, prolong the functional period of leaves, and increase the rice yield.

\section{References}

Alhagdow M.F., Mounet L., Gilbert A. et al.: Silencing of the mitochondrial ascorbate synthesizing enzyme l-galactono1,4-lactone dehydrogenase affects plant and fruit development in tomato. - Plant Physiol. 145: 1408-1422, 2007.

Barth C., Moeder W., Klessig D.F., Conklin P.L.: The timing of senescence and response to pathogens is altered in the ascorbate-deficient Arabidopsis mutant vitamin c-1. - Plant Physiol. 134: 1784-1792, 2004.

Bienert G.P., Schjoerring J.K., Jahn T.P.: Membrane transport of hydrogen peroxide. - BBA-Biomembranes 1758: 994-1003, 2006.

Buchanan-Wollaston V., Page T., Harrison E. et al.: Comparative transcriptome analysis reveals significant differences in gene expression and signalling pathways between developmental and dark/starvation-induced senescence in Arabidopsis. Plant J. 42: 567-585, 2005.

Chiang C.M., Chen C.C., Chen S.P. et al.: Overexpression of the ascorbate peroxidase gene from eggplant and sponge gourd enhances flood tolerance in transgenic Arabidopsis. - J. Plant Res. 130: 373-386, 2017.

De Pinto M.C., Locato V., De Gara L. et al.: Redox regulation in plant programmed cell death. - Plant Cell Environ. 35: 234244, 2012.

Fang M., Zhou Z., Zhou X. et al.: Overexpression of OsFTL10 induces early flowering and improves drought tolerance in Oryza sativa L. - PeerJ 7:e6422, 2019.

Gallie D.R.: The role of 1-ascorbic acid recycling in responding to environmental stress and in promoting plant growth. J. Exp. Bot. 64: 433-443, 2013.

Gest N., Gautier H., Stevens R.: Ascorbate as seen through plant evolution: the rise of a successful molecule. - J. Exp. Bot. 64: 33-53, 2013.

Gregersen P.L., Culetic A., Boschian L., Krupinska K.: Plant senescence and crop productivity. - Plant Mol. Biol. 82: 603-622, 2013.

Guerreiro J.R.L., Kamel A.H., Sales M.G.F.: FIA potentiometric system based on periodate polymeric membrane sensors for the assessment of ascorbic acid in commercial drinks. - Food Chem. 120: 934-939, 2010.

Hideg E., Schreiber U.: Parallel assessment of ROS formation and photosynthesis in leaves by fluorescence imaging. Photosynth. Res. 92: 103-108, 2007.

Iizumi T., Ramankutty N.: Changes in yield variability of major crops for 1981-2010 explained by climate change. - Environ. Res. Lett. 11: 34003, 2016.

Jiang H.W., Li M.R., Liang N.T. et al.: Molecular cloning and function analysis of the stay green gene in rice. - Plant J. 52: 197-209, 2007.

Jing H.C., Nam H.G.: Leaf senescence in plants: from model plants to crops, still so many unknowns. - J. Integr. Plant Biol. 54: 514-515, 2012.

Lee S.H., Sakuraba Y., Lee T. et al: : Mutation of Oryza sativa CORONATINE INSENSITIVE $1 \mathrm{~b}(\mathrm{OSCOI1b)}$ delays leaf senescence. - J. Integr. Plant Biol. 57: 562-576, 2015.
Li S., Wang J., Yu Y. et al:: D27E mutation of VTC1 impairs the interaction with CSN5B and enhances ascorbic acid biosynthesis and seedling growth in Arabidopsis. - Plant Mol. Biol. 92: 473-482, 2016.

Li Z., Wakao S., Fischer B.B., Niyogi K.K.: Sensing and responding to excess light. - Annu. Rev. Plant Biol. 60: 239-260, 2009.

Liang C.Z., Wang Y.Q., Zhu Y.N. et al.: OsNAP connects abscisic acid and leaf senescence by fine-tuning abscisic acid biosynthesis and directly targeting senescence-associated genes in rice. - P. Natl. Acad. Sci. USA 111: 10013-10018, 2014.

Lichtenthaler H.K.: Chlorophylls and carotenoids: Pigments of photosynthetic biomembranes. - Method. Enzymol. 148: 350382, 1987.

Lim P.O., Kim H.J., Nam H.G.: Leaf senescence. - Annu. Rev. Plant Biol. 58: 115-136, 2007.

Liu Y., Ren D., Pike S. et al.: Chloroplast-generated reactive oxygen species are involved in hypersensitive response-like cell death mediated by a mitogen-activated protein kinase cascade. - Plant J. 51: 941-954, 2007.

Liu Y.H., Peng X.X., Li M.Q. et al.: [Degradation of ribulose1,5-bisphosphate carboxylase/oxygenase in rice leaves under oxidative stress induced by methyl viologen.] - Acta Photophysiol. Sin. 26: 481-486, 2000. [In Chinese with English abstract]

Livak K.J., Schmittgen T.D.: Analysis of relative gene expression data using real-time quantitative PCR and the $2^{-\Delta \Delta \mathrm{CT}}$ method. Methods 25: 402-408, 2001.

Mae T.: Physiological nitrogen efficiency in rice: Nitrogen utilization, photosynthesis and yield potential. - Plant Soil 196: 201-210, 1997.

Mekki E.D., Hussien H.A., Salem H.: Role of glutathione, ascorbic acid and $\alpha$-tocopherol in alleviation of drought stress in cotton plants. - Int. J. ChemTech Res. 8: 1573-1581, 2015.

Mittler R., Vanderauwera S., Gollery M., Van Breugesem F.: Reactive oxygen gene network of plants. - Trends Plant Sci. 9: 490-498, 2004.

Müller-Moulé P., Havaux M., Niyogi K.K.: Zeaxanthin deficiency enhances the high light sensitivity of an ascorbate-deficient mutant of Arabidopsis. - Plant Physiol. 133: 748-760, 2003.

Nam H.G.: The molecular genetic analysis of leaf senescence. Curr. Opin. Biotech. 8: 200-207, 1997.

Ou Z.Y., Peng C.L., Lin G.Z. et al.: Regulation of senescence in rice flag leaf growing in the field by excitation pressure. Acta Bot. Sin. 45: 929-935, 2003.

Pavet V., Olmos E., Kiddle G. et al.: Ascorbic acid deficiency activates cell death and disease resistance responses in Arabidopsis. - Plant Physiol. 139: 1291-1303, 2005.

Potters G., Horemans N., Jansen M.A.K.: The cellular redox state in plant stress biology - a charging concept. - Plant Physiol. Bioch. 48: 292-300, 2010.

Ren L., Zhang D., Chen G. et al.: Transcriptomic profiling revealed the regulatory mechanism of Arabidopsis seedlings response to oxidative stress from cryopreservation. - Plant Cell Rep. 34: 2161-2178, 2015.

Saha M.R., Hasan R., Akter R. et al.: In vitro free radical scavenging activity of methanol extract of the leaves of Mimusops elengi Linn. - Bangl. J. Vet. Med. 6: 197-202, 2008.

Smirnoff N., Wheeler G.L.: Ascorbic acid in plants: biosynthesis and function. - Crit. Rev. Biochem. Mol. Biol. 35: 291-314, 2000.

Tóth S.Z., Puthur J.T., Nagy V., Garab G.: Experimental evidence for ascorbate-dependent electron transport in leaves with inactive oxygen-evolving complexes. - Plant Physiol. 149: 
1568-1578, 2009.

Van Breusegem F., Dat J.F.: Reactive oxygen species in plant cell death. - Plant Physiol. 141: 384-390, 2006.

Woo H.R., Kim H.J., Nam H.G., Lim P.O.: Plant leaf senescence and death-regulation by multiple layers of control and implications for aging in general. - J. Cell Sci. 126: 4823-4833, 2013.

Xu S.B., Yu H.T., Yan L.F., Wang T.: Integrated proteomic and cytological study of rice endosperms at the storage phase. J. Proteome Res. 9: 4906-4918, 2010.

Yang X., Gong P., Li K. et al.: A single cytosine deletion in the OSPLS1 gene encoding vacuolar-type $\mathrm{H}^{+}$-ATPase subunit A1 leads to premature leaf senescence and seed dormancy in rice.J. Exp. Bot. 67: 2761-2776, 2016.

Yu L., Liu Y.H., Lu L. et al.: Ascorbic acid deficiency leads to increased grain chalkiness in transgenic rice for suppressed of L-GalLDH. - J. Plant Physiol. 211: 13-26, 2017.

Yu L., Liu Y.H., Tong J.H. et al.: Reduced grain chalkiness and its possible physiological mechanism in transgenic rice overexpressing L-GalLDH. - Crop J. 3: 125-134, 2015.

Zeng D.D., Yang C.C., Qin R. et al.: A guanine insert in OsBBS1, leads to early leaf senescence and salt stress sensitivity in rice (Oryza sativa L.). - Plant Cell Rep. 37: 933-946, 2018.

Zhang C., Ouyang B., Yang C. et al.: Reducing AsA leads to leaf lesion and defence response in knock-down of the AsA biosynthetic enzyme GDP-D-mannose pyrophosphorylase gene in tomato plant. - PLoS ONE 8: e61987, 2013.

Zhang C.J., Chu H.J., Chen G.X. et al.: Photosynthetic and biochemical activities in flag leaves of a newly developed superhigh-yield hybrid rice (Oryza sativa) and its parents during the reproductive stage. - J. Plant Res. 120: 209-217, 2007.

Zhang Q.L., Wei Y.X., Peng C.L.: Effects of endogenous ascorbic acid on resistance to high-temperature stress in excised rice leaves. - Photosynthetica 56: 1453-1458, 2018.

(C) The authors. This is an open access article distributed under the terms of the Creative Commons BY-NC-ND Licence. 